Rev. Econ. Design 3, 129-135 (1998)

Review
of Economic
Design
(c) Springer-Verlag 1998

\title{
Equitable nature of core allocations in atomless economies
}

\author{
Farhad Hüsseinov \\ Department of Economics, Bilkent University, TR-06533 Bilkent, Ankara, Turkey \\ Received: 7 March 1995 / Accepted: 3 March 1997
}

\begin{abstract}
The purpose of this paper is to prove the equal treatment property for the $\star$-core allocations of an atomless economy without any condition on the data of economy. This result prompts the same property for the core allocations.
\end{abstract}

JEL classification: C60, C70, D51

Key words: Atomless exchange economy, core, equal treatment, measurable correspondence

\section{Introduction}

Following Green (1972) we shall say that an economy possesses the equal treatment property, if for each allocation from the core, agents of the same type (i.e. with the same endowments and tastes) receive the equally desired commodity bundles. If the property stated above is fulfilled for a given allocation, we shall say that allocation possesses equal treatment property.

Equal treatment property was first established apparently by Debreu and Scarf (1963) for finite economy. A slight and immediate generalization of this result (see Green 1972) asserts that finite economy in which the numbers of agents of the same type have a greatest common divisor not less than two possesses the equal treatment property.

Obviously, an arbitrary competitive allocation treats the agents of the same type equally. This property is therefore necessary for an economy to have the core equivalence property, i.e. the property of coincidence of the core and the set of competitive equilibria. On the other hand, the proof of core equivalence theorem is often carried out by establishing some variants of the equal treatment property (see e.g., Gabszewicz and Mertens 1971 and Green 1972). 
We are considering atomless pure exchange economies. Aumann's Core Equivalence Theorem entails that if an atomless economy satisfies some conditions (such as continuity, desirability of preferences, strict positivity of total endowments etc.) then it possesses the equal treatment property. It is shown here that in atomless economies "equal treatment property holds coalitionally" i.e. similar members of two identical coalitions receive equally desired bundles at $\star$-core allocations (see Definition in Sect. 3) without any condition on the data of economy (Theorem 1). From that result validity of equal treatment property for $\star$-core allocations is derived. From this in turn, equal treatment property for atomless economies satisfying minimal conditions (continuity and local insatiability of preferences) is derived. Notice that the situation for the finite economies is opposite to the situation in atomless economies. Namely, it was shown by Green (1972) that if the greatest common divisor of the numbers of the agents of the same type is equal to one, then for almost all initial assignments (in the sense of Lebesgue measure on the space of all initial assignments) equal treatment property doesn't hold.

\section{The model}

We consider the well-known continual model of exchange of $\ell$ different commodities between continuum of agents $\mathscr{E}=\left\{(T, \Sigma, \nu),\left(X, \omega, \succ_{t}\right)\right\}$, where $(T, \Sigma, \nu)$ is a finite atomless measure space of agents, $X$ is correspondence of $T$ into the commodity space $\mathbf{R}^{l}$ describing the consumption sets of agents, $\omega$ is a $\nu$ integrable function of $T$ into $\mathbf{R}^{l}$, describing the distribution of initial endowments and for each $t \in T, \succ_{t}$ is a preference relation of agent $t$, which is an arbitrary irreflexive binary relation on $X(t)$. We suppose that the family of preferences $\succ_{t}(t \in T)$ is measurable, i.e. the set $\left\{(t, x, y) \in T \times \mathbf{R}^{l} \times \mathbf{R}^{l}: x \succ_{t} y\right\}$ belongs to the product $\Sigma$-algebra $\Sigma_{\nu} \otimes \mathscr{B}^{l} \otimes \mathscr{B}^{l}$, where $\Sigma_{\nu}$ denotes the completion of $\Sigma$ relative to $\nu, \mathscr{B}^{l}$ the $\Sigma$-algebra of the Borel subsets in $\mathbf{R}^{l}$. It will be assumed also that correspondence $X$ is $\left(\Sigma, \mathscr{B}^{l}\right)$-measurable.

For an arbitrary two vectors $x, y \in X(t)$ we will denote $x \sim_{t} y$, if neither $x \succ_{t} y$ nor $y \succ_{t} x$. It should be noted that the relation $\sim_{t}$ is reflexive, symmetric but not transitive relation and hence it is not an equivalence relation. For a $\nu$ integrable function $x: T \rightarrow \mathbf{R}^{l}$, notations $\int x$ and $\int_{E} x$ will mean $\nu$-integral of $x$ over $T$ and over $E \in \Sigma$, respectively; so $\int x=\int_{T} x$.

An assignment is a $\nu$-integrable function $x: T \rightarrow \mathbf{R}^{l}$ such that $x(t) \in X(t)$ for $\nu$-almost all $t \in T$. An allocation is an assignment $x$ satisfying condition $\int x=\int \omega$. A coalition is a $\Sigma$-measurable subset of $T$ with positive $\nu$-measure. An assignment $y$ dominates an allocation $x$ via coalition $E$, if 1) $y(t) \succ_{t} x(t)$ for $t \in E$, 2) $\int_{E} y=\int_{E} \omega$. The set of all undominated allocations is called a core of economy $\mathscr{E}$ and is denoted by $C(\mathscr{E})$. 


\section{Equal treatment property of core allocations}

Definition (Shitovitz, 1973, p. 479). An assignment $\boldsymbol{y} \star$-dominates an allocation $x$ via coalition $S$ ( $S$ is said to $\star$-block $x$ ), if (1) $\nu(R)>0$, where $R=\{t \in$ $\left.S: y(t) \succ_{t} x(t)\right\}$ (2) for almost all $t \in S \backslash R, y(t)=x(t)$ and (3) $\int_{S} y=\int_{S} \omega$. The set of all $\star$-undominated allocations of economy $\mathscr{E}$ will be called $\star$-core of economy $\mathscr{E}$ and denoted by $C^{\star}(\mathscr{E})$.

Theorem 1. Let $\mathscr{E}=\left\{(T, \Sigma, \nu),\left(X(t), \omega(t), \succ_{t}\right), t \in T\right\}$ be an atomless pure exchange economy. Let $T_{1}, T_{2} \in \Sigma$ be disjoint coalitions such that there exists a one-to-one measure preserving mapping $\varphi: T_{1} \rightarrow T_{2}$ such that $X(t)=$ $X(\varphi(t)), \omega(t)=\omega(\varphi(t))$ and $\succ_{t}=\succ_{\varphi(t)}$ for $t \in T_{1}$. Then for $x(\cdot) \in C^{\star}(\mathscr{E})$

$$
x(t) \sim_{t} x(\varphi(t)) \text { for almost all } t \in T_{1} .
$$

Proof. Suppose that for an allocation $x(\cdot) \in C^{\star}(\mathscr{E})$ relation (1) is not satisfied. In this case, by passing if necessary, to the subsets of $T_{1}$ and $T_{2}$, we can see that there exists a coalition $A_{1} \in \Sigma$, such that

$$
x(\varphi(t)) \succ_{t} x(t) \quad \text { for } \quad t \in A_{1} .
$$

Put $m(S)=\left(\int_{S} x, \int_{S} \omega, \nu(S)\right)$ for $S \in \Sigma$. Obviously $m$ is $(2 l+1)$-vector measure on $(T, \Sigma)$. By Liapunov's Theorem there exist $B_{1} \subset A_{1}$ and $B_{2} \subset A_{2}$, where $A_{2}=\varphi\left(A_{1}\right)$, such that

$$
m\left(B_{1}\right)=\frac{1}{4} m\left(A_{1}\right) \text { and } m\left(B_{2}\right)=\frac{1}{4} m\left(A_{2}\right) .
$$

Two cases are possible:

1) $B_{2}=\varphi\left(B_{1}\right)$ up to a set of $\nu$ measure zero. In this case again by Liapunov's Theorem there exists $C_{2} \subset A_{2} \backslash B_{2}$ such that

$$
m\left(C_{2}\right)=\frac{1}{3} m\left(A_{2} \backslash B_{2}\right)
$$

It follows from (3) and (4) that

$$
m\left(C_{2}\right)=\frac{1}{4} m\left(A_{2}\right) .
$$

Clearly the sets $B_{1}$ and $C_{1}=\varphi^{-1}\left(C_{2}\right)$ are disjoint (up to a set of $\nu$-measure zero). Denote by $D_{1}=B_{1} \cup C_{1}$ and

$$
y(t)=\left\{\begin{array}{lll}
x(t) & \text { for } & t \in T \backslash C_{1}, \\
x(\varphi(t)) & \text { for } & t \in C_{1} .
\end{array}\right.
$$

By Liapunov's Theorem there exists $E \subset T \backslash A$, where $A=A_{1} \cup A_{2}$ such that

$$
m(E)=\frac{1}{4} m(T \backslash A)
$$

Putting $S=E \cup D_{1}$ we have from (5) and (6) 


$$
\begin{aligned}
\int_{S} \omega & =\int_{E} \omega+\int_{B_{1}} \omega+\int_{C_{1}} \omega=\frac{1}{4} \int_{T \backslash A} \omega+\frac{1}{4} \int_{A_{1}} \omega+\int_{C_{2}} \omega \\
& =\frac{1}{4} \int_{T \backslash A} \omega+\frac{1}{4} \int_{A_{1}} \omega+\frac{1}{4} \int_{A_{2}} \omega=\frac{1}{4} \int_{T} \omega .
\end{aligned}
$$

Similarly, $\int_{S} y=\frac{1}{4} \int_{T} x$. The last two relations together with equality $\int_{T} x=$ $\int_{T} w$ give $\int_{S} y=\int_{S} \omega$.

It is clear that $y(t)=x(t)$ for $t \in B_{1} \cup E$, and $y(t)=x(\varphi(t)) \succ_{t} x(t)$ for $t \in C_{1}$, by (2), i.e. $y(\cdot) \star$-dominates $x(\cdot)$ via coalition $S$.

2) $B_{2} \neq \varphi\left(B_{1}\right)$ (up to a set of $\nu$-measure zero). Put $G_{2}=B_{2} \backslash \varphi\left(B_{1}\right)$ and $G_{1}=$ $\varphi^{-1}\left(G_{2}\right)$. Obviously $G_{1} \cap B_{1}=\emptyset$. Put $H=B_{1} \cup G_{1} \cup K$, where $K=B_{2} \cap \varphi\left(B_{1}\right)$ and

$$
y(t)=\left\{\begin{array}{lll}
x(\varphi(t)) & \text { for } & t \in G_{1}, \\
x(t) & \text { for } & t \in T \backslash G_{1},
\end{array}\right.
$$

and let $E$ be chosen as in the case 1). Put $S=E \cup M$. We assert that $y(\cdot)$ $\star$-dominates $x(\cdot)$ via coalition $S$. Indeed,

$$
\begin{aligned}
\int_{S} y & =\int_{E} y+\int_{H} y=\int_{E} y+\int_{B_{1}} y+\int_{G_{1}} y+\int_{K} y \\
& =\int_{E} x+\int_{B_{1}} x+\int_{G_{1}} x(\varphi(t))+\int_{K} x=\frac{1}{4} \int_{T / A} x+\frac{1}{4} \int_{A_{1}} x+\int_{G_{2}} x+\int_{K} x \\
& =\frac{1}{4} \int_{T / A_{2}} x+\int_{B_{2}} x=\frac{1}{4} \int_{T \backslash A_{2}} x+\frac{1}{4} \int_{A_{2}} x=\frac{1}{4} \int_{T} x
\end{aligned}
$$

Similarly, $\int_{S} \omega=\frac{1}{4} \int_{S} \omega$.

The last two relations together with equality $\int_{T} x=\int_{T} \omega$ yield $\int_{S} y=\int_{S} \omega$. On the other hand

$$
y(t)=x(t) \text { for } t \in S \backslash G_{1}, \quad \text { and } \quad y(t)=x(\varphi(t)) \succ_{t} x(t) \text { for } t \in G_{1} .
$$

Moreover $\nu\left(G_{1}\right)>0$. Therefore assignment $y(\cdot) \star$-dominates $x(\cdot)$ via coalition $S$. Thus we have seen that in both cases there exists assignment $y(\cdot) \star$-dominating allocation $x(\cdot)$, which contradicts to $x(\cdot) \in C^{\star}(\mathscr{E})$.

Remark 1. Since the Liapunov Convexity Theorem fails for an arbitrary infinite dimensional space (see e.g. Diestel and Uhl (1977)) the above proof of Theorem 1 is not valid for the case of infinite dimensional commodity space. Nevertheless we conjecture that Theorem 1 holds for an arbitrary infinite dimensional commodity space, but as yet have been unable to prove this conjecture.

Theorem 2 from Kingman and Robertson (1968) allows to state that if initial endowments of agents are not "too dispersed" then Theorem 1 holds for each not "too dispersed" *-core allocation. We consider a function $z: T \rightarrow E$, where $E$ is a locally convex space, as not "too dispersed" if the following set of integrable functions $\left\{\langle z, f\rangle(t)=\langle z(t), f\rangle, t \in T \mid f \in E^{\prime}\right\}$, where $E^{\prime}$ is a topological dual of $E$, is thin. For the definition of last term see Kingman and Robertson (1968). 
If $(X, \mathscr{C}, \mu)$ and $(Y, \mathscr{B}, \nu)$ are measure spaces, a point isomorphism between $X$ and $Y$ is a one-to-one mapping $J$ from almost all of $X$ on almost all of $Y$ such that $E \in \mathscr{C}$ if and only if $F=J(E) \in \mathscr{B}$, and then $\mu(E)=\nu(F)$. A finite measure space is said to be normal measure space if it is point isomorphic to a finite interval with Lebesgue measure. The original definition of normal measure space given by Halmos P. and J. von Neumann (1942) is different from the one given above. The equivalence of the two definitions was established in their Geometric Isomorphism Theorem (p. 339, Theorem 2).

Corollary. Assume that in atomless economy $\mathscr{E}=\{(\mathscr{T}, \Sigma, \nu),(\mathscr{C}, \ldots, \succ)\}$, where $(T, \Sigma, \nu)$ is a normal space, $T_{0}$ is any coalition of agents with the same initial data, i.e. $\left(X(t), \omega(t), \succ_{t}\right)=(X, \omega, \succ)$ for $t \in T_{0}$ and $x(\cdot) \in C^{\star}(\mathscr{E})$. Then $x\left(t_{1}\right) \sim x\left(t_{2}\right)$ for $t_{1}, t_{2} \in T_{0}^{\prime}$, where $T_{0}^{\prime} \subset T_{0}$ and $\nu\left(T_{0}^{\prime}\right)=\nu\left(T_{0}\right)$.

Proof. Suppose not. Then there exist disjoint coalitions $A_{1}, A_{2}$, such that

$$
x\left(t_{2}\right) \succ x\left(t_{1}\right), \quad \forall t_{1} \in A_{1}, \quad \forall t_{2} \in A_{2} .
$$

Since measure $\nu$ is atomless, we can assume that $\nu\left(A_{1}\right)=\nu\left(A_{2}\right)$ (see Halmos, 1974 , p. 174, Exercise 2). Moreover, we can assume that $A_{1}, A_{2}$ are Borel subsets of $T$ in the sense of Halmos-Neumann (1942). By Lemmas 1 and 3 from HalmosNeumann (1942) $A_{1}, A_{2}$ are normal spaces. Applying the Geometric Isomorphism Theorem we obtain a measure preserving mapping $\varphi: A_{1} \rightarrow A_{2}$. Then by Theorem $1 x(t) \sim x(\varphi(t))$, for each $t \in A_{1}$. Contradiction between this and relations (7) proves the corollary.

Observe that in Theorem 1 no assumption other than that of non-atomicity of measure $\nu$ is made on the data of the model. Moreover, if some assumptions on the data of economy are made then the core of economy coincides with the set of Walras allocations and therefore for such models equitability property holds. Now we will derive this property directly from Theorem 1 thereby dispensing with price mechanism. First we give the following lemma, which strengthens the corresponding result (Lemma 4) from (Shitovitz 1973); in particular, desirability of preferences $\succ_{t}$ is not assumed in this lemma.

Lemma. Let $\succ_{t}(t \in T)$ be continuous and locally non-satiable in economy $\mathscr{E}$. Then, if an assignment $y \star$-dominates $x$ via coalition $E$, then there exists an assignment $z$ which dominates $x$ via the same coalition $E$. Therefore under considered assumptions $\star$-core coincides with the core of economy $\mathscr{E}$, i.e. $C^{\star}(\mathscr{E})=\mathscr{C}(\mathscr{E})$.

For the Proof of Lemma see Appendix. It will be seen that this proof, unlike that of Lemma 4 in Shitovitz (1973) is direct and doesn't use any results concerning exchange models.

Theorem 2. Let in economy $\mathscr{E}$ preferences $\succ_{t}(t \in T)$ be continuous and locally insatiable. Then assertion of Theorem 1 holds for an arbitrary allocation $x$ from the core $C(\mathscr{E})$ of economy $\mathscr{E}$. 
Remark 2. All the above results easily generalize to the case of economies with production. Suppose $\mathscr{E}=\{(\mathscr{T}, \Sigma, \nu),(\mathscr{X}, \omega, \succ), \mathscr{Y}\}$ is a production economy described in Hildenbrand (1968). Recall that here $Y$ is the (production) correspondence from $\Sigma$ into $\mathbf{R}^{l}$ satisfying relevant conditions (see Hildenbrand 1968). In this case Definition of $\star$-domination should be given as follows: an assignment $y \star$-dominates an allocation $x$ via coalition $S$, if (1) $\nu(R)>0$, where $R=\left\{t \in S: y(t) \succ_{t} x(t)\right\}$, (2) for almost all $t \in S \backslash R, y(t)=x(t)$ and (3) $\int_{S} y \in \int_{S} \omega+Y(S)$. In Theorem 1 mapping $\varphi: T_{1} \rightarrow T_{2}$ must satisfy in addition, assumption $Y(E)=Y(\varphi(E))$ for each $E \in \Sigma, E \in T_{1}$.

\section{Appendix}

The Appendix is devoted to the establishment of one proposition concerning integral of correspondence and a proof of the Lemma, which asserts coincidence of the $\star$-core and core of economy. Note that this Lemma under stronger assumptions was proved in by Shitovitz (1973).

We use the standard notations. Symbol $\|\cdot\|$ stands for Euclid norm in $\mathbf{R}^{l}$ and $B_{\delta}(x)$ for an open ball in $\mathbf{R}^{l}$ with center at $x$ and radius $\delta$. For a subset $A$ of $\mathbf{R}^{l}$ denote by $\bar{A}$ and $\partial A$ its closure and boundary, respectively. For a point $a \in \mathbf{R}^{l}$ and a subset $A \subset R^{l}$ denote by dist $(a, A)$ distance between $a$ and $A$. For a correspondence $X: T \rightarrow 2^{\mathbf{R}^{l}}$, where $(T, \Sigma, \nu)$ is a finite measure space, the set of all measurable selectors of $X$ denote by $L_{X}$. By $\bar{X}: T \rightarrow 2^{\mathbf{R}^{1}}$ denote a correspondence defined by $\bar{X}(t)=\overline{X(t)}$ for $t \in T$.

Proposition. Let $(T, \Sigma, \nu)$ be a finite measure space and let $X: T \rightarrow 2^{\mathbf{R}^{\mathbf{1}}}$ be a non-empty open measurable corespondence. Let $y \in L_{\bar{X}}$ be integrable and such that $y(t) \in X(t)$ for $t \in T_{0}$, where $T_{0} \in \Sigma, \nu\left(T_{0}\right)>0$. Then $\int y \in \int X$, i.e. there exists integrable function $x \in L_{X}$ such that $\int y=\int x$.

Proof. Without loss of generality suppose that $\nu(T)=1$. Let $y \in L_{\bar{X}}$ satisfies the assumptions of Proposition. Denote $y_{0}=\int_{T_{0}} y$ and consider a function $\delta(t)=$ $\operatorname{dist}(y(t), \partial X(t))$ for $t \in T_{0}$. Obviously, $\delta(t)$ is a measurable positive function. Denote $\delta_{0}=\int_{T_{0}} \delta(t) d \nu(t)$. It is easily seen that

$$
y_{0}+B_{\delta_{0}}(0) \subset \int_{T_{0}} X .
$$

Consider a correspondence $Z(t)=X(t) \cap B_{\delta_{0}}(y(t))$ for $t \in T_{1}=T \backslash T_{0}$. Since $y(t) \in \bar{X}(t), Z(t) \neq \phi$ for $t \in T_{1}$. It follows from the theory of measurable correspondences (see e.g. Ioffe and Tikhomirov 1974), that $Z$ is a measurable correspondence and hence by Mesurable Choice Theorem there exists $x_{1} \in L_{\left.Z\right|_{T_{1}}}$. Obviously, $x_{1}$ is a $\nu$-integrable function and $\left\|\int_{T_{1}} x_{1}-\int_{T_{1}} y\right\|<\delta_{0}$.

Denote $u=-\int_{T_{1}} x_{1}+\int_{T_{1}} y$. By inclusion (1) $y_{0}+u \in \int_{T_{0}} X$. Therefore there exists $x_{0} \in L_{\left.X\right|_{0}}$ such that $\int_{T_{0}} x_{0}=y_{0}+u$. Put 


$$
x(t)= \begin{cases}x_{0}(t), & \text { if } \quad t \in T_{0}, \\ x_{1}(t), & \text { if } \quad t \in T_{1} .\end{cases}
$$

Then $x \in L_{X}$ and $\int x=\int y$.

QED.

Proof of Lemma. Put $X(t)=\left\{x \in \mathbf{R}_{+}^{l}: x \succ_{t} x(t)\right\}$ for $t \in E$. It follows from the local insatiability of preferences $\succ_{t}(t \in T)$ that $y(t) \in \bar{X}(t)$ for $t \in E$. Applying Proposition we get $\int_{E} y \in \int_{E} X$, i.e. there exists $z_{0} \in L_{X}$ such that $\int_{E} z_{0}=\int_{E} y$.

Put

$$
z(t)=\left\{\begin{array}{lll}
z_{0}(t) & \text { for } & t \in E \\
\omega(t) & \text { for } & t \in E
\end{array}\right.
$$

Then evidently $z$ dominates $x$ via coaliton $E$.

\section{References}

1. Aumann, R.J. (1964) Markets with a confinuum of traders. Econometrica 32: 39-50

2. Debreu, G., Scarf, H. (1963) A limit theorem on the core of an economy. Int. Econ. Rev. 4: 235-246

3. Diestel, J., Uhl, J.J. (1979) Vector Measures. Springer, Berlin, Heidelberg, New York

4. Gabszewicz, J.J., Mertens, J.-F. (1971) An equivalence theorem for the core of an economy whose atoms are not "too" big. Econometrica 39: 713-721

5. Green, J.R. (1972) On the inequitable nature of core allocations. J. Econ. Theory 4: 132-143

6. Halmos, P.R., Neumann, J. (1942) Operator methods in classical mechanics, II. Ann. Math. 43: $332-350$

7. Halmos, P.R. (1974) Measure Theory. Springer, Berlin, Heidelberg, New York

8. Hildenbrand, W. (1968) The core of an economy with a measure space of economic agents. Rev. Econ. Studies 35: 443-452

9. Ioffe, A.D., Tikhomirov, V.M. (1974) Theory of Extremal Problems. Nauka, Moscow

10. Kingman, J.F.C., Robertson, A.P. (1968) On a Theorem of Lyapunov. J. London Math. Soc. 43: 347-351

11. Liapunov, A. (1940) Sur les functions-vecteurs complement additives. Izv. Akad. Nauk. SSSR, Ser. Math. 4: 465-478

12. Shitovitz, B. (1973) Oligopoly in markets with a continuum of traders. Econometrica 41: 467501 